\title{
Metastatic Colorectal Cancer: Optimizing Treatment with Anti-EGFR Monoclonal Antibody
}

\author{
Ka-On Lam*, Victor Ho-Fun Lee \\ Department of Clinical Oncology, Li Ka Shing Faculty of Medicine, The University of Hong Kong, Hong Kong, China. \\ Email: *lamkaon@hku.hk
}

Received September $30^{\text {th }}, 2012$; revised October $31^{\text {st }}, 2012$; accepted November $10^{\text {th }}, 2012$

\begin{abstract}
Colorectal cancer remains a major cause of morbidity and mortality worldwide despite substantial improvement in the standard of care. Although surgical resection in selected patients may potentially be curative, systemic therapy is the only choice of treatment for most patients with metastatic colorectal cancer. Anti-epidermal growth factor receptor (anti-EGFR) monoclonal antibody has established its role in the systemic therapy of metastatic colorectal cancer through multiple well-designed clinical trials and yet, the optimal use of anti-EGFR monoclonal antibody is undefined. This article serves to review the available evidence for anti-EGFR monoclonal antibodies and to speculate optimal strategy for their uses.
\end{abstract}

Keywords: Colorectal Cancer; Epidermal Growth Factor Receptor; Cetuximab; Panitumumab; Strategy

\section{Introduction}

Colorectal cancer is the third most common cancer in men and the second in women worldwide. Every year, about 608,000 deaths are estimated, making colorectal cancer the fourth most common cause of cancer-related death [1]. Among patients with colorectal cancer, around $40 \%$ of patients present with or will eventually develop distant metastasis. The principal modality of treatment for patients with metastatic disease is systemic therapy and approved sole or combination options include: 5fluorouracil, capecitabine, oxaliplatin, irinotecan, bevacizumab, cetuximab and panitumumab. Among all these options, only the anti-epidermal growth factor receptor (anti-EGFR) monoclonal antibodies, cetuximab and panitumumab, have proven efficacy in early as well as late lines of therapy.

\subsection{Epidermal Growth Factor Receptor (EGFR)}

The ErbB family of receptors, which is also known as the human epidermal growth factor receptor (HER), plays a critical role in carcinogenesis. Being the first receptor identified in the family, EGFR is also known as the type I receptor tyrosine kinase or ErbB1/HER1. The other member receptors in this family are ErbB2 (HER2/neu), ErbB3 (HER3) and ErbB4 (HER4) [2-4].

The EGFR is located on the cell surface and is struc-

"Corresponding author. turally composed of an extracellular ligand-binding domain, a transmembrane segment and an intracellular tyrosine kinase domain. A variety of ligands, including epidermal growth factor, transforming growth factor- $\alpha$, amphiregulin and epiregulin, is able to bind to EGFR. Upon ligand binding, EGFR forms homo- or heterodimers with other members of the ErbB family resulting in autophosphorylation of the intracellular domain and subsequent activation of downstream intracellular signaling cascade $[5,6]$.

The Ras/Raf/ mitogen-activated protein kinase (MAPK), the PI3K/AKT and the Jak2/Stat3 pathways are major downstream signaling pathways that are crucial for cancer cell proliferation, survival, invasion, metastasis and neo-angiogenesis [7].

\subsection{Anti-EGFR Monoclonal Antibody}

Monoclonal antibody directed at the EGFR was first studied on mice in the 1980's [8,9] but not until February 2004 when the first anti-EGFR monoclonal antibody was approved for treating metastatic colorectal cancer in human. Monoclonal antibodies, such as cetuximab and panitumumab, bind to the extracellular domain of EGFR and, hence block the ligand-binding induced receptor dimerization as well as tyrosine kinase activation [10]. In addition, cetuximab, being a chimeric antibody, is able to elicit antibody-dependent cellular cytotoxicity against cancer cells [11,12] 


\section{Anti-EGFR Antibodies in Key Phase III Studies}

KRAS is a GTP-binding protein which plays a crucial role in the downstream intracellular signaling cascade of various receptor tyrosine kinases including the EGFR. Activating mutation of KRAS occurs in $\sim 40 \%$ of patients with colorectal cancer. Mutations in the KRAS oncogene will lead to constitutive activation of the KRAS protein independent of the upstream regulation and thus, may affect the efficacy of anti-EGFR antibodies. In the following section, evidence from the key phase III clinical trials using anti-EGFR antibodies will be evaluated, with special attention to their differential activities in KRAS wild-type and mutant tumors (Table 1).

\subsection{Anti-EGFR Antibodies in the First-Line Setting}

\subsubsection{The Crystal Study}

The CRYSTAL study was the first to demonstrate overall survival benefit with the use of cetuximab in the first-line treatment of metastatic colorectal cancer. It was a phase III open-label, randomized, multi-center study that compared cetuximab plus FOLFIRI and FOLFIRI alone [13]. 1198 patients with EGFR-expressing adenocarcinoma were enrolled. After a median follow-up of around 30 months, patients in the cetuximab-FOLFIRI arm had significantly prolonged progression-free survival (8.9 vs 8.0 months; HR, 0.85; $p=0.048$ ). The high proportion of patients received post-study chemotherapy in

Table 1. Clinical efficacy of anti-EGFR antibody in phase III clinical trials: stratified according to KRAS status.

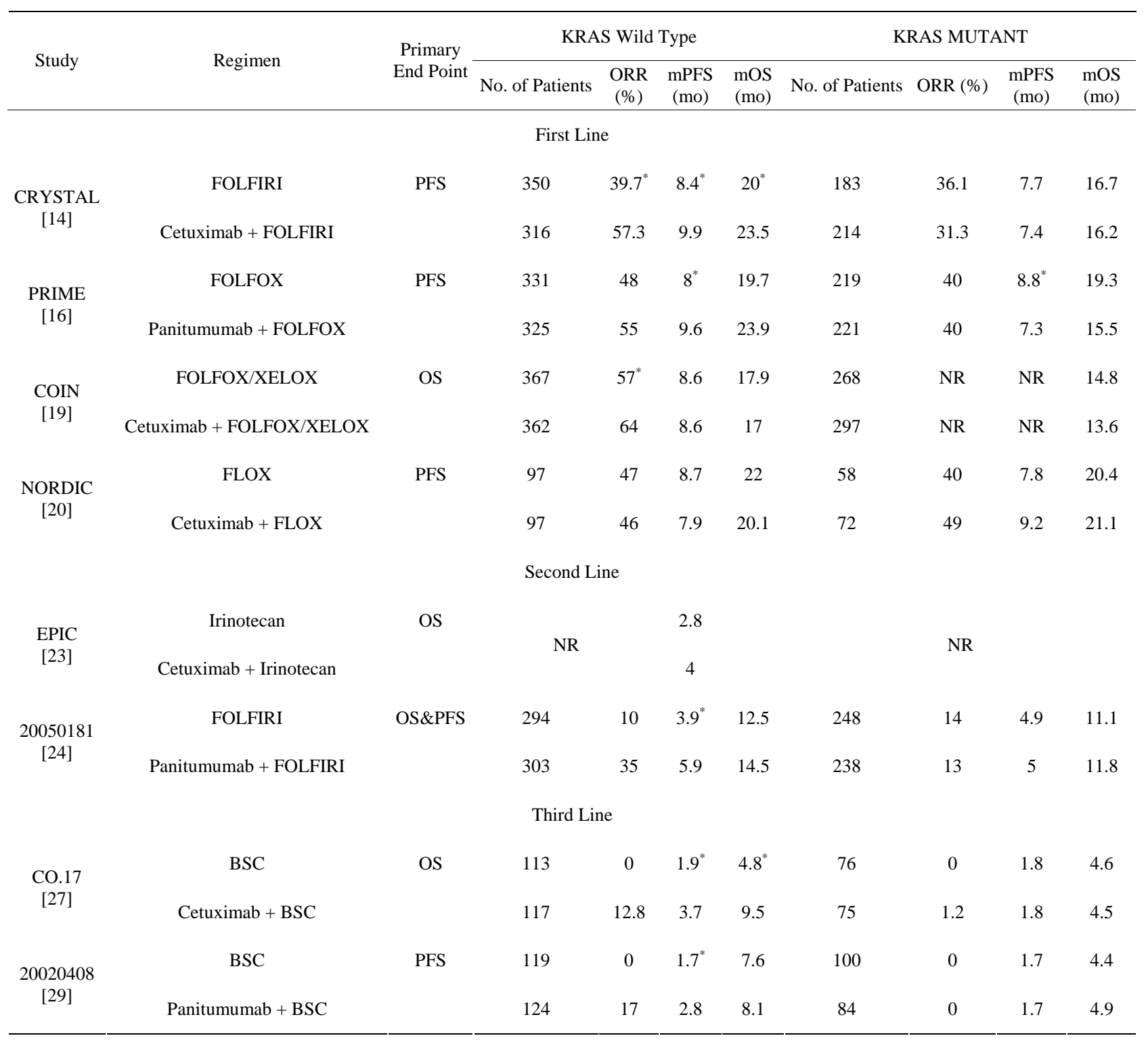

ORR: overall response rate; mPFS: median progression-free survival; mOS: median overall survival; mo: months. * statistical significant. 
both arms might explain why no statistically significant difference in overall survival was achieved. Indeed, antiEGFR antibody was given to $25.4 \%$ and $6.2 \%$ of patients in the FOLFIRI and cetuximab-FOLFIRI arm, respectively, after progression. Increasing grade of skin toxicity correlates with longer progression-free survival in cetuximab-treated patients.

Further retrospective analyses confirmed KRAS mutational status as a strong predictor of clinical outcomes in patients treated with cetuximab. The addition of cetuximab to FOLFIRI in patients with KRAS wild-type disease resulted in significant improvements in overall survival (23.5 vs 20.0 months; HR, 0.796; $\mathrm{p}=0.0093$ ), progression-free survival (9.9 vs 8.4 months; HR, 0.696; $\mathrm{p}=$ 0.0012 ) and response rate $(57.3 \%$ vs $39.7 \%$; OR, 2.069 ; $\mathrm{p}<0.001$ ) [14] In the pooled analysis of the CRYSTAL and OPUS trials, similar magnitude of benefit were demonstrated for overall survival (HR, $0.81 ; \mathrm{p}=0.0062$ ), progression-free survival (HR, 0.66 ; $p<0.001)$ and response rate (OR, 2.16; $p<0.0001)$ [15]. In both analyses, BRAF status was shown to be prognostic rather than predictive [14,15].

\subsubsection{The PRIME Study}

A phase III, open-label, randomized, multi-center study that evaluated the role of panitumumab with FOLFOX4 in the first-line treatment of metastatic colorectal cancer [16]. While both the PRIME study and the CRYSTAL study recruited unselected patients with respect to the KRAS status, a prospective analysis on treatment outcomes according to KRAS status was included only in the former. $93 \%$ of the 1,183 randomly assigned patients had KRAS status available. KRAS wild-type tumor comprised $60 \%$ of the whole study population. Although not stratified in the random assignment, this proportion was preserved in both the experimental and control arm. Within the KRAS wild-type stratum, progression-free survival, the primary endpoint of the study, was significantly improved from 8.0 months to 9.6 months [HR, $0.80 ; p=0.02$ ]. The overall survival, the secondary endpoint, was increased from 19.7 months to 23.9 months but no statistical significant difference was reached.

The overall survival result should be interpreted with data on post-progression treatment: $8 \%$ and $18 \%$ of patients in the panitumumab-FOLFOX4 arm and FOLFOX4 arm received anti-EGFR antibodies, respectively. More patients in the FOLFOX4 arm (62\%) than those in the panitumumab-FOLFOX4 arm (53\%) received subsequent chemotherapy. Bevacizumab was also given in $12 \%$ $15 \%$ of patients.

Similar to the result of the biomarker analysis in the OPUS study [17], the PRIME study has shown that KRAS status was predictive of panitumumab efficacy. In the KRAS mutant stratum, patients who received pani- tumumab has reduced progression-free survival [HR 1.29; $\mathrm{p}=0.02$ ] and overall survival [HR, 1.24; $\mathrm{p}=0.068$ ]. However, subsequent analysis of adverse events as well as dose intensity did not reveal a plausible explanation for the detrimental effect of panitumumab on KRAS mutant tumor $[16,18]$.

\subsubsection{The MRC COIN Study}

The MRC COIN study was the largest phase III, randomized, multicenter study that compared chemotherapy with or without cetuximab on overall survival as the primary endpoint [19]. A prospective analysis of KRAS status on treatment effect and molecular analysis of BRAF and NRAS were also performed. One thousand six hundred and thirty patients were randomized and tumor samples from 1316 patients were available for molecular analysis. Oxaliplatin was combined with either infusional 5-fluorourail or capecitabine as the chemotherapy backbone. Contrary to previous results with antiEGFR antibodies, the overall survival and progressionfree survival were not improved with the use of cetuximab even in KRAS wild-type tumor. The only significant finding was an improvement of overall response rate from $57 \%$ to $64 \%$ [OR, 1.35; $p=0.049$ ].

Interestingly, this largest trial on cetuximab raised the critical question of what is the appropriate chemotherapy partner with cetuximab. The addition of cetuximab to capecitabine-based chemotherapy resulted in increased toxicity, especially grade 3 or above diarrhoea (30\%), which mandated a protocol amendment for dose reduction of capecitabine from $1000 \mathrm{mg} / \mathrm{m}^{2}$ to $850 \mathrm{mg} / \mathrm{m}^{2}$. Of patients taking cetuximab, numerically more treatmentrelated deaths were reported in the subgroup that received capecitabine-based therapy. As such the dose intensity of capecitabine-based therapy reduced significantly from $85 \%$ in the control arm to $79 \%$ in the cetuximab arm [p $=0.0021]$.

Subsequent analysis also showed that the only group of patients that may gain benefit from cetuximab is 1) KRAS wild-type tumors; 2) Limited distribution of metastatic disease; and 3) Treated with infusional fluorouracil. [HR, 0.55; $\mathrm{p}=0.011]$.

Irrespective of treatment received, somatic mutation status correlated with overall survival: 14.4 months in KRAS mutant, 8.8 months in BRAF mutant and 20.1 months in all wild-type.

\subsubsection{The NODIC VII Study}

The NODIC VII study concluded that cetuximab did not add significant benefit in the first-line treatment of metastatic colorectal cancer [20]. It was a 3-arm phase III, randomized, multicenter study that investigated the benefit of cetuximab and compared continuous versus intermittent regimen. The chemotherapy backbone were ox- 
aliplatin and bolus 5-fluorouracil, also known as the Nordic FLOX, which is a standard regimen in the Nordic countries [21]. The impact of KRAS status was also included after a protocol amendment but no sample size re-estimation was performed. Five-hundreds and sixtysix patients were randomized to ensure a greater than $80 \%$ power to detect an increase in progression-free survival from 7 to 10 months. Knowing that this magnitude of benefit has never been achieved with any of the anti-EGFR antibodies, the conclusion of no significant difference is not entirely unexpected. In all the study arms, in both the intention-to-treat and the KRAS wildtype subset, the progression-free survival, overall survival and the response rate were almost identical. Unexpectedly, a trend towards improved progression-free survival was observed in KRAS mutant tumor. Further analysis that addressed the probable differential response in KRAS mutant tumors did not show any difference in efficacy for patients with codon 13 (G13D) and codon 12 mutations treated with cetuximab.

\subsection{Anti-EGFR Antibodies in the Second-Line Setting}

\subsubsection{The EPIC Study}

A phase III open-label, randomized study that was designed to determine whether the addition of cetuximab to irinotecan as second-line therapy would prolong survival [22]. Patients were eligible if they were irinotecan-naïve and have failed both oxaliplatin and fluoropyrimidine in their first-line treatment. Although prior bevacizumab was allowed (bevacizumab was given in $13 \%$ of patients in both arms), previous exposure to anti-EGFR therapies were excluded.

The study failed to meet its primary end point of improvement in overall survival. This was probably due to the fact that a substantial proportion (46.9\%) of patients in the irinotecan alone arm subsequently received cetuximab-based therapy. Compared with patients treated with irinotecan alone, those treated with cetuximab and irinotecan had significantly longer progression-free survival [4.0 vs 2.6 months, HR: 0.692, $\mathrm{p}<0.0001]$ and higher overall response rate [ $16.4 \%$ vs $4.2 \%$, $p<0.0001$ ].

Despite longer treatment duration and more treatmentrelated toxicities, cetuximab and irinotecan were significantly more effective in maintaining overall quality of life.

The KRAS mutation status was retrospectively analyzed from $23 \%$ of patients but the progression-free survival, overall survival and response rate did not differ significantly in the KRAS wild-type tumor [23].

\subsubsection{The 20050181 Study}

A phase III, open-label, randomized, multicenter study that compared the efficacy of panitumumab plus FOLFIRI versus FOLFIRI alone [24]. Patients were eligible if they have failed fluoropyrimidine-based therapy in the firstline setting. Prior treatments with irinotecan and antiEGFR therapy were not allowed. Unlike the EPIC study, failure of oxaliplatin treatment is not required and KRAS status was prospectively analyzed.

In the 1186 patients that were randomized, ninety-one percent of them had specimen available for KRAS status test. In the KRAS wild-type subgroup, the addition of panitumumab to FOLFIRI significantly prolonged the progression-free survival from 3.9 months to 5.9 months [HR, 0.73; $\mathrm{p}=0.004]$ and improved the response rate from $10 \%$ to $35 \%$ [p $<0.001$ ]. However, the difference in overall survival failed to reach statistical significance [14.5 months versus 12.5 months; HR, 0.85; $\mathrm{p}=0.12$ ]. Contrary to the result of PRIME study, panitumumab did not appear to have detrimental effect on response rate, progression-free survival and overall survival in the KRAS mutant subgroup.

\subsection{Anti-EGFR Antibodies in the Third-Line Setting}

\subsubsection{The BOND Study}

In the BOND study, 329 patients who progressed during or within 3 months after treatment with an irinotecanbased regimen were randomized in 2:1 ratio to receive either cetuximab and irinotecan or cetuximab monotherapy [25]. The number of prior chemotherapy was not restricted but around $80 \%$ of patients received study treatment at the third-line or beyond setting. The response rate was significantly different, being $22.9 \%$ in the combination arm and $10.8 \%$ in the cetuximab monotherapy arm [p $=0.007]$. The time to progression was also significantly prolonged from 1.5 months to 4.1 months [HR: $0.54, \mathrm{p}<0.001$ ]. The overall survival was not different significantly as patients in the cetuximab monotherapy arm were allowed to receive irinotecan upon disease progression.

\subsubsection{The NCIC CO.17 Study}

A phase III randomized, multicenter study that compared single agent cetuximab to best supportive care in patients who have failed or contraindicated to fluoropyrimidine, oxaliplatin and irinotecan [26]. The primary end point was overall survival and a total of 572 patients were randomized. Cetuximab prolonged the median overall survival from 4.6 months to 6.1 months [HR: 0.77, p = 0.005]. The result did reflect the unconfounded benefit of cetuximab monotherapy since only a small proportion of patients went on to receive any post-progression therapy.

Interestingly, an unplanned subgroup analysis demonstrated that the grade of rash in patients receiving cetuxi- 
mab strongly correlated with overall survival, being 2.6 months in patients with no rash, 4.8 months in patients with grade 1 rash and 8.4 months in patients with grade 2 rash [p $<0.001]$.

A retrospective analysis on KRAS status was subsequently performed on $69 \%$ of patients. In the KRAS wild-type subgroup, the progression-free survival, overall survival and response rate were significantly improved with cetuximab over best supportive care. The response rate to cetuximab monotherapy was $12.8 \%$. In the KRAS mutant group, clinical outcomes were not significantly different between the cetuximab arm and best supportive care arm $[27,28]$.

\subsubsection{The 20020408 Study}

A phase III randomized, multicenter study on the use of panitumumab versus best supportive care in chemotherapy-refractory patients [29]. Unlike the NCIC CO.17 study, eligible patients have to fail 2 to 3 lines of chemotherapy, and to be exposed to a defined dose intensity of both oxaliplatin and irinotecan. A pre-planned analysis on KRAS status was included.

463 patients were randomized and the progression-free survival was significantly prolonged in the panitumumab arm [8 weeks vs 7.3 weeks; HR, 0.54; $\mathrm{p}<0.0001$ ]. The overall survival was similar between the two study arms but was likely the result of the high rate and rapidity of cross-over after progression in the supportive-care arm. The objective response rate was $10 \%$ with panitumumab monotherapy.

KRAS status was available for $92 \%$ of patients. In patients with KRAS wild-type tumor, the progression free survival, the primary end point of the study, was significantly increased [12.3 weeks vs 7.3 weeks; HR, 0.45]. The overall survival differed significantly in neither of the KRAS subgroups.

\section{Evolving Strategies with Anti-EGFR Antibody}

\subsection{Anti-EGFR Antibody Concurrent with Anti-VEGF Antibody}

Preclinical data has shown a close relationship of VEGF and EGFR signaling cascade and that the combine of both anti-VEGF and anti-EGFR therapy would be a biologically plausible and attractive strategy [30-33].

\subsubsection{The BOND 2 Study}

A phase II randomized study to evaluate the benefit of bevacizumab added to cetuximab and irinotecan or cetuximab alone in patients who were irinotecan-refractory [34]. The median numbers of regimens before study entry was 3 in both arms. The primary end point was time to tumor progression and 83 patients were enrolled. In the bevacizumab, cetuximab and irinotecan arm, the time to tumor progression was 7.3 months and the response rate was $37 \%$. Even in the bevacizumab and cetuximab arm, the time to tumor progression reached 4.9 months and the response rate $20 \%$.

\subsubsection{The CAIRO 2 Study}

A phase III randomized multicenter study that recruited 755 previously untreated patients to study the benefit of cetuximab added to a regimen of bevacizumab, capecitabine and oxaliplatin [35]. The primary end point was progression-free survival and KRAS was evaluated as a predictor of outcome. The addition of cetuximab has led to a significantly shorter progression-free survival [9.4 months vs 10.7 months; HR, 1.22; $\mathrm{p}=0.01$ ] and inferior quality of life while the overall survival and response rate were not significantly different. In the KRAS mutant subgroup, the progression-free survival was shortened with the addition of cetuximab.

\subsubsection{PACCE Study}

A US community-based, randomized phase IIIB study that evaluated the efficacy and safety of bevacizumab and chemotherapy, either oxaliplatin or irinotecan, with or without panitumumab in the first-line setting [36]. A total of 823 and 230 patients were randomly assigned to the oxaliplatin and irinotecan cohorts, respectively. Panitumumab was discontinued after a planned interim analysis of 812 oxaliplatin patients that showed worse efficacy in the panitumumab arm. In the final analysis, panitumumab was shown to reduce the progression-free survival from 11.4 months in the control arm to 10.0 months [HR, 1.27]. Subgroup analysis of the KRAS status also showed adverse outcomes in both wild-type and mutant subgroups with panitumumab. While the exact mechanism of this unexpected finding was not clearly explained, the higher rate of toxicity in the panitumumab arm might be the main culprit. In the oxaliplatin cohort, an excess of grade $3 / 4$ adverse events, including skin toxicity, diarrhea, infection and pulmonary embolism, was observed.

Although the efficacy of concurrent cetuximab and bevacizumab seems encouraging in non-first line setting, concrete evidence from the two large phase III studies has suggested that this combination should not be applied outside clinical trial setting.

\subsection{Anti-EGFR Antibody Maintenance}

Although patients in most clinical trials are required to continue treatment till disease progression, in daily practice patients usually prefer "drug holiday" after initial disease control. The perceived benefit of a chemother- 
apy-free interval is the improved quality of life and reduced side effect from ongoing therapy. However, both the OPTIMOX2 [37] and the COIN study [38] have shown that a complete stop of chemotherapy would result in a shorter duration of disease control. The improved toxicity profile has thus made target therapy an attractive option.

In the NORDIC study [20], patients randomized to the intermittent-chemotherapy arm stopped the FLOX chemotherapy after 16 weeks but continued cetuximab as maintenance therapy. FLOX chemotherapy was then reintroduced while continuing cetuximab upon disease progression. With this approach, the clinical outcomes including the overall survival was similar to those patients on the continuous-chemotherapy arm. This is an early piece of evidence that maintenance therapy with cetuximab may be a feasible strategy and that further studies are warranted.

\subsection{Anti-EGFR Antibody beyond Progression}

Unlike the benefit of using bevacizumab beyond disease progression [39], data regarding the use of anti-EGFR antibodies in this setting is relatively limited. In the above mentioned study, the rate of post-study anti-EGFR antibodies therapy was $6 \%-12 \%[13,16,20,24]$. The post-hoc exploratory analysis of the EPIC study suggested a potential clinical benefit of using cetuximab beyond disease progression [22]. Patients in the cetuximab-irinotecan arm who went on to receive cetuximab in the post-study therapy has a median survival of 16.2 months [95\% CI, 12.8 to 27.4 months] while those that did not receive any post-study therapy and those that received post-study therapy without cetuximab had median survival of 6.31 months [95\% CI, 5.3 to 7.1 months] and 13.0 months [95\% CI, 11.6 to 13.9 months]. Taking into account the selection bias inherent to this unplanned analysis, the result should be regarded as hypothesisgenerating rather than practice changing and further studies are warranted.

\subsection{Anti-EGFR Antibody Rechallenge}

Based on the scientific data the KRAS status largely remains the same during tumor progression [40] and that secondary KRAS mutations do not occur during antiEGFR therapy [41], rechallenging patients with antiEGFR antibody is another biologically plausible strategy in late-line therapy against KRAS wild-type tumor.

A phase II prospective study was set up to evaluate the activity of rechallenge with cetuximab-based therapy [42]. Patients were retreated, after a new line of chemotherapy, with the same cetuximab and irinotecan-based regimen that they have benefited before. 39 patients were enrolled and the median number of prior therapies was four. The overall response rate was 53.8\% including 2 complete responses. The median progression-free survival was 6.6 months.

Two smaller studies have investigated the efficacy of panitumumab monotherapy after cetuximab failure. The PANERB study was a single-arm, prospective, community-based study that recruited 39 patients with KRAS wild-type tumor [43]. For patients that have responded to cetuximab-based therapy, subsequent panitumumab monotherapy was able to achieve a response rate of $54.5 \%$ and stable disease in $18.2 \%$ of patients. In case of cetuximab resistance, the response rate was $7.7 \%$ only. However, a second single-arm study that recruited 20 patients with unequivocal progression while on cetuximab showed somewhat inferior result [44]. No objective response was demonstrated and the stable disease rate was $45 \%$ with a median duration of only 1.7 months.

Definitive evidence is lacking for the use of antiEGFR antibody after cetuximab failure but it is suggested a small subset of patients with KRAS wild-type tumor who had initial response to cetuximab-based therapy might benefit from this approach.

\subsection{Anti-EGFR Antibody and Anti-VEGF Antibody as Sequential Therapy}

With the use of chemotherapy in metastatic colorectal cancer, it was shown that the exact sequence of chemotherapeutic agents used did not affect outcomes [45] as long as patients were exposed to all active agents [46]. However, there is no strong evidence to support the same principle in the use of anti- EGFR and anti- VEGF antibodies. In fact, all clinical studies that support anti-EGFR and anti-VEFG antibody in non-first line setting were performed in era before the widespread use of these target therapies in the first-line setting [22,24,47]. In preclinical studies, cancer cells may become more dependent on VEGF pathway when they acquired resistance to anti-EGFR therapy [48-50] and a sequence-specific clinical benefit with these therapies would be a reasonable assumption.

In the 20050181 study, around $20 \%$ of patients in each study arm have received bevacizumab before study entry. Patients were stratified according to previous bevacizumab exposure and subgroup analysis on both progression-free survival and overall survival favored the addition of panitumumab irrespective of previous bevacizumab exposure [24]. On the other hand, the effect of prior bevacizumab exposure on the efficacy of subsequent cetuximab treatment was explored in a small series of patients but prior bevacizumab exposure were associated with a significantly inferior outcome during subsequent cetuximab treatment [51].

There is no phase III study published yet to investigation specifically the clinical effect of sequential use of 
anti-EGFR antibody and anti-VEGF antibody. The socalled FIRE3 study by the German AIO group may provide insight in this respect [52]. The study compared the efficacy for cetuximab plus FOLFIRI versus bevacizumab plus FOLFIRI in the first-line treatment. The second-line treatment was not randomized but data was captured. Only the result in the KRAS mutant subgroup has been reported while the result in the KRAS wild-type subgroup is still awaited.

\section{Conclusions}

It took almost two decades for anti-EGFR monoclonal antibody from bench to bedside. The ability to select patients according to KRAS mutational status has revolutionalized the field of personalized therapy in the treatment of metastatic colorectal cancer. In the majority of clinical trials, anti-EGFR monoclonal antibody has proven efficacy and enhanced benefit is seen in KRAS wild-type tumor. The few studies that reported "unconventional" results should be viewed as an opportunity to further refine current treatment strategy in the context of previously-established evidence.

With the best available evidence, the following can be concluded:

\section{Selection of patients:}

Anti-EGFR antibodies should only be used in patients with KRAS wild-type tumor [14,16,19,24,27,29]. The consistent improvement in response rate across all lines of treatment favors their use especially in patient with potentially resectable disease as well as patients with high tumor burden and significant tumor-related symptoms.

Selection of chemotherapy partners:

Irinotecan would be a robust choice [13,22,24,25]. The evidence of combining an anti-EGFR antibody with oxaliplatin is less clear and sometimes conflicting [16,17, $19,20]$. The use of infusional 5FU is preferred to bolus 5FU or capecitabine as clinical benefit was demonstrated only with the former $[13,16,24]$ while increased toxicity and even reduced clinical efficacy were seen with the latter $[19,20]$.

\section{Combining with anti-VEGF antibodies:}

Concurrent administration of anti-EGFR and antiVEGF antibodies should be prohibited in routine clinical practice $[35,36]$. There is no recommendation on the sequential use of these antibodies and more studies are encouraged in the future.

Selection of novel strategy:

With the limited number of available agents against metastatic colorectal cancer, maximizing benefit of each agent is of utmost importance. The use of anti-EGFR antibodies in maintenance and rechallenge settings are both promising. However, their use beyond progression appears to have limited role.

In the years to come, improved knowledge of the mechanism of resistance and interaction with other target therapies as well as chemotherapeutic agents would help define an optimal strategy in the treatment of metastatic colorectal cancer with anti-EGFR monoclonal antibody.

\section{REFERENCES}

[1] http://globocan.iarc.fr/factsheets/cancers/colorectal.asp

[2] Y. Yarden and M. X. Sliwkowski, "Untangling the ErbB Signalling Network," Nature Reviews Molecular Cell Biology, Vol. 2, No. 2, pp. 127-137. doi:10.1038/35052073

[3] L. N. Klapper, H. Waterman, M. Sela and Y. Yarden, "Tumor-Inhibitory Antibodies to HER-2/ErbB-2 May Act by Recruiting c-Cbl and Enhancing Ubiquitination of HER-2," Cancer Research, Vol. 60, No. 13, 2000, pp. 3384-3388.

[4] M. A. Olayioye, R. M. Neve, H. A. Lane and N. E. Hynes, "The ErbB Signaling Network: Receptor Heterodimerization in Development and Cancer," The EMBO Journal, Vol. 19, No. 13, 2000, pp. 3159-3167. doi:10.1093/emboj/19.13.3159

[5] A. Citri and Y. Yarden, "EGF-ERBB Signalling: Towards the Systems Level," Nature Reviews Molecular Cell Biology, Vol. 7, No. 7, 2006, pp. 505-516. doi:10.1038/nrm1962

[6] N. E. Hynes and H. A. Lane, "ERBB receptors and Cancer: The Complexity of Targeted Inhibitors," Nature Reviews Cancer, Vol. 5, No. 5, 2005, pp. 341-354. doi:10.1038/nrc1609

[7] J. Mendelsohn and J. Baselga, "Status of Epidermal Growth Factor Receptor Antagonists in the Biology and Treatment of Cancer,” Journal of Clinical Oncology, Vol. 21, No. 14, 2003, pp. 2787-2799. doi:10.1200/JCO.2003.01.504

[8] J. Sato, T. Kawamoto, A. Le, J. Mendelsohn, J. Polikoff and G. Sato, "Biological Effects in Vitro of Monoclonal Antibodies to Human Epidermal Growth Factor Receptors," Molecular Biology and Medicine, Vol. 1, No. 5, 1983, pp. 511-529.

[9] H. Masui, T. Kawamoto, J. D. Sato, B. Wolf, G. Sato and J. Mendelsohn, "Growth Inhibition of Human Tumor Cells in Athymic Mice by Anti-Epidermal Growth Factor Receptor Monoclonal Antibodies," Cancer Research, Vol. 44, No. 3, 1984, pp. 1002-1007.

[10] S. Li, K. R. Schmitz, P. D. Jeffrey, J. J. W. Wiltzius, P. Kussie and K. M. Ferguson, "Structural Basis for Inhibition of the Epidermal Growth Factor Receptor by Cetuximab,” Cancer Cell, Vol. 7, No. 4, 2005, pp. 301-311. doi:10.1016/j.ccr.2005.03.003

[11] H. Kimura, K. Sakai, T. Arao, T. Shimoyama, T. Tamura and K. Nishio, "Antibody-Dependent Cellular Cytotoxicity of Cetuximab Against Tumor Cells with Wild-Type or Mutant Epidermal Growth Factor Receptor," Cancer Science, Vol. 98, No. 8, 2007, pp. 1275-1280. doi:10.1111/j.1349-7006.2007.00510.x 
[12] Y. Kawaguchi, K. Kono, K. Mimura, H. Sugai, H. Akaike and H. Fujii, "Cetuximab Induce Antibody-Dependent Cellular Cytotoxicity against EGFR-Expressing Esophageal Squamous Cell Carcinoma," International Journal of Cancer, Vol. 120, No. 4, 2007, pp. 781-787. doi:10.1002/ijc.22370

[13] E. Van Cutsem, C.-H. Köhne, E. Hitre, J. Zaluski, C.-R. C. Chien, A. Makhson, et al., "Cetuximab and Chemotherapy as Initial Treatment for Metastatic Colorectal Cancer," New England Journal of Medicine, Vol. 360, No. 14, 2009, pp. 1408-1417. doi:10.1056/NEJMoa0805019

[14] E. Van Cutsem, C.-H. Köhne, I. Láng, G. Folprecht, M. P. Nowacki, S. Cascinu, et al., "Cetuximab plus Irinotecan, Fluorouracil, and Leucovorin as First-Line Treatment for Metastatic Colorectal Cancer: Updated Analysis of Overall Survival According to Tumor KRAS and BRAF Mutation Status," Journal of Clinical Oncology, Vol. 29, No. 15, 2011, pp. 2011-2019. doi:10.1200/JCO.2010.33.5091

[15] C. Bokemeyer, E. Van Cutsem, P. Rougier, F. Ciardiello, S. Heeger, M. Schlichting, et al., "Addition of Cetuximab to Chemotherapy as First-Line Treatment for KRAS Wild-Type Metastatic Colorectal Cancer: Pooled Analysis of the Crystal and OPUS Randomised Clinical Trials," European Journal of Cancer, Vol. 48, No. 10, 2012, pp. 1466-1475. doi:10.1016/j.ejca.2012.02.057

[16] J.-Y. Douillard, S. Siena, J. Cassidy, J. Tabernero, R. Burkes, M. Barugel, et al., "Randomized, Phase III Trial of Panitumumab with Infusional Fluorouracil, Leucovorin, and Oxaliplatin (FOLFOX4) versus FOLFOX4 Alone as First-Line Treatment in Patients with Previously Untreated Metastatic Colorectal Cancer: The PRIME Study,” Journal of Clinical Oncology, Vol. 28, No. 31, 2010, pp. 4697-4705. doi:10.1200/JCO.2009.27.4860

[17] C. Bokemeyer, I. Bondarenko, J. T. Hartmann, F. de Braud, G. Schuch, A. Zubel, et al., "Efficacy According to Biomarker Status of Cetuximab Plus FOLFOX-4 as first-Line Treatment for Metastatic Colorectal Cancer: the OPUS Study,” Annals of Oncology, Vol. 22, No. 7, 2011, pp. 1535-1546. doi:10.1093/annonc/mdq632

[18] R. G. Amado, M. Wolf, M. Peeters, E. Van Cutsem, S. Siena, D. J. Freeman, et al., "Wild-Type KRAS Is Required for Panitumumab Efficacy in Patients with Metastatic Colorectal Cancer," Journal of Clinical Oncology, Vol. 26, No. 10, 2008, pp. 1626-1634. doi:10.1200/JCO.2007.14.7116

[19] T. S. Maughan, R. A. Adams, C. G. Smith, A. M. Meade, M. T. Seymour, R. H. Wilson, et al., "Addition of Cetuximab to Oxaliplatin-Based First-Line Combination Chemotherapy for Treatment of Advanced Colorectal Cancer: Results of the Randomised Phase 3 MRC COIN Trial,” The Lancet, Vol. 377, No. 9783, 2011, pp. 2103-2114. doi:10.1016/S0140-6736(11)60613-2

[20] K. M. Tveit, T. Guren, B. Glimelius, P. Pfeiffer, H. Sorbye, S. Pyrhonen, et al., "Phase III Trial of Cetuximab with Continuous or Intermittent Fluorouracil, Leucovorin, and Oxaliplatin (Nordic FLOX) versus FLOX Alone in First-Line Treatment of Metastatic Colorectal Cancer: The NORDIC-VII Study,” Journal of Clinical Oncology, Vol. 30, No. 15, 2012, pp. 1755-1762. doi:10.1200/JCO.2011.38.0915
[21] H. Sørbye, B. Glimelius, Å. Berglund, T. Fokstuen, K. M. Tveit, M. Brændengen, et al., "Multicenter Phase II Study of Nordic Fluorouracil and Folinic Acid Bolus Schedule Combined with Oxaliplatin as First-Line Treatment of Metastatic Colorectal Cancer,” Journal of Clinical Oncology, Vol. 22, No. 1, 2004, pp. 31-38. doi:10.1200/jco.2004.05.188

[22] A. F. Sobrero, J. Maurel, L. Fehrenbacher, W. Scheithauer, Y. A. Abubakr, M. P. Lutz, et al., "EPIC: Phase III Trial of Cetuximab plus Irinotecan after Fluoropyrimidine and Oxaliplatin Failure in Patients with Metastatic Colorectal Cancer," Journal of Clinical Oncology, Vol. 26, No. 14, 2008, pp. 2311-2319. doi:10.1200/JCO.2007.13.1193

[23] C. Langer, J. Kopit and M. Awad, “Analysis of KRAS Mutations in Patients with Metastatic Colorectal Cancer Receiving Cetuximab in Combination with Irinotecan: Results from the EPIC Trial,” Annals of Oncology, Vol. 26, Suppl. 8, 2008, p. viii133.

[24] M. Peeters, T. J. Price, A. Cervantes, A. F. Sobrero, M. Ducreux, Y. Hotko, et al., "Randomized Phase III Study of Panitumumab with Fluorouracil, Leucovorin, and Irinotecan (FOLFIRI) Compared with FOLFIRI Alone as Second-Line Treatment in Patients with Metastatic Colorectal Cancer,” Journal of Clinical Oncology, Vol. 28, No. 31, 2010, pp. 4706-4713. doi:10.1200/JCO.2009.27.6055

[25] D. Cunningham, Y. Humblet, S. Siena, D. Khayat, H. Bleiberg, A. Santoro, et al., "Cetuximab Monotherapy and Cetuximab plus Irinotecan in Irinotecan-Refractory Metastatic Colorectal Cancer," New England Journal of Medicine, Vol. 351, No. 4, 2004, pp. 337-345. doi:10.1056/NEJMoa033025

[26] D. J. Jonker, C. J. O’Callaghan, C. S. Karapetis, J. R. Zalcberg, D. Tu, H.-J. Au, et al., "Cetuximab for the Treatment of Colorectal Cancer," New England Journal of Medicine, Vol. 357, No. 20, 2007, pp. 2040-2048. doi:10.1056/NEJMoa071834

[27] C. S. Karapetis, S. Khambata-Ford, D. J. Jonker, C. J. O’Callaghan, D. Tu, N. C. Tebbutt, et al., "K-Ras Mutations and Benefit from Cetuximab in Advanced Colorectal Cancer," New England Journal of Medicine, Vol. 359, No. 17, 2008, pp. 1757-1765. doi:10.1056/NEJMoa0804385

[28] H.-J. Au, C. S. Karapetis, C. J. O’Callaghan, D. Tu, M. J. Moore, J. R. Zalcberg, et al., "Health-Related Quality of Life in Patients with Advanced Colorectal Cancer Treated with Cetuximab: Overall and KRAS-Specific Results of the NCIC CTG and AGITG CO.17 Trial,” Journal of Clinical Oncology, Vol. 27, No. 11, 2009, pp. 1822-1828. doi:10.1200/JCO.2008.19.6048

[29] E. Van Cutsem, M. Peeters, S. Siena, Y. Humblet, A. Hendlisz, B. Neyns, et al., "Open-Label Phase III Trial of Panitumumab plus Best Supportive Care Compared with Best Supportive Care Alone in Patients with Chemotherapy-Refractory Metastatic Colorectal Cancer," Journal of Clinical Oncology, Vol. 25, No. 13, 2007, pp. 1658-1664. doi:10.1200/JCO.2006.08.1620

[30] F. Ciardiello, R. Bianco, V. Damiano, G. Fontanini, R. Caputo, G. Pomatico, et al., "Antiangiogenic and Antitumor Activity of Anti-Epidermal Growth Factor Recep- 
tor C225 Monoclonal Antibody in Combination with Vascular Endothelial Growth Factor Antisense Oligonucleotide in Human GEO Colon Cancer Cells," Clinical Cancer Research, Vol. 6, No. 9, 2000, pp. 3739-3747.

[31] R. M. Shaheen, S. A. Ahmad, W. Liu, N. Reinmuth, Y. D. Jung, W. W. Tseng, et al., "Inhibited Growth of Colon Cancer Carcinomatosis by Antibodies to Vascular Endothelial and Epidermal Growth Factor Receptors," British journal of cancer, Vol. 85, No. 4, 2001, pp. 584-589. doi:10.1054/bjoc.2001.1936

[32] Y. D. Jung, P. F. Mansfield, M. Akagi, A. Takeda, W. Liu, C. D. Bucana, et al., "Effects of Combination AntiVascular Endothelial Growth Factor Receptor and AntiEpidermal Growth Factor Receptor Therapies on the Growth of Gastric Cancer in a Nude Mouse Model,” European Journal of Cancer, Vol. 38, No. 8, 2002, pp. 1133-1140. doi:10.1016/S0959-8049(02)00013-8

[33] J. R. Tonra, D. S. Deevi, E. Corcoran, H. Li, S. Wang, F. E. Carrick, et al., "Synergistic Antitumor Effects of Combined Epidermal Growth Factor Receptor and Vascular Endothelial Growth Factor Receptor-2 Targeted Therapy,” Clinical Cancer Research, Vol. 12, No. 7, 2006, pp. 2197-2207. doi:10.1158/1078-0432.CCR-05-1682

[34] L. B. Saltz, H.-J. Lenz, H. L. Kindler, H. S. Hochster, S. Wadler, P. M. Hoff, et al., "Randomized Phase II Trial of Cetuximab, Bevacizumab, and Irinotecan Compared with Cetuximab and Bevacizumab Alone in Irinotecan-Refractory Colorectal Cancer: The BOND-2 Study," Journal of Clinical Oncology, Vol. 25, No. 29, 2007, pp. 45574561. doi:10.1200/JCO.2007.12.0949

[35] J. Tol, M. Koopman, A. Cats, C. J. Rodenburg, G. J. M. Creemers, J. G. Schrama, et al., "Chemotherapy, Bevacizumab, and Cetuximab in Metastatic Colorectal Cancer," New England Journal of Medicine, Vol. 360, No. 6, 2009, pp. 563-572. doi:10.1056/NEJMoa0808268

[36] J. R. Hecht, E. Mitchell, T. Chidiac, C. Scroggin, C. Hagenstad, D. Spigel, et al., “A Randomized Phase IIIB Trial of Chemotherapy, Bevacizumab, and Panitumumab Compared with Chemotherapy and Bevacizumab Alone for Metastatic Colorectal Cancer,” Journal of Clinical Oncology, Vol. 27, No. 5, 2009, pp. 672-680. doi:10.1200/JCO.2008.19.8135

[37] B. Chibaudel, F. Maindrault-Goebel, G. Lledo, L. Mineur, T. André, M. Bennamoun, et al., "Can Chemotherapy Be Discontinued in Unresectable Metastatic Colorectal Cancer? The GERCOR OPTIMOX2 Study," Journal of Clinical Oncology, Vol. 27, No. 34, 2009, pp. 5727-5733. doi:10.1200/JCO.2009.23.4344

[38] R. A. Adams, A. M. Meade, M. T. Seymour, R. H. Wilson, A. Madi, D. Fisher, et al., "Intermittent versus Continuous Oxaliplatin and Fluoropyrimidine Combination Chemotherapy for First-Line Treatment of Advanced Colorectal Cancer: Results of the Randomised Phase 3 MRC COIN Trial," the Lancet Oncology, Vol. 12, No. 7, 2011, pp. 642-653. doi:10.1016/S1470-2045(11)70102-4

[39] A. Grothey, M. M. Sugrue, D. M. Purdie, W. Dong, D. Sargent, E. Hedrick, et al., "Bevacizumab Beyond First Progression Is Associated with Prolonged Overall Survival in Metastatic Colorectal Cancer: Results from a
Large Observational Cohort Study (BRiTE),” Journal of Clinical Oncology, Vol. 26, No. 33, 2008, pp. 5326-5334. doi:10.1200/JCO.2008.16.3212

[40] D. Santini, F. Loupakis, B. Vincenzi, I. Floriani, I. Stasi, E. Canestrari, et al., "High Concordance of KRAS Status between Primary Colorectal Tumors and Related Metastatic Sites: Implications for Clinical Practice,” The Oncologist, Vol. 13, No. 12, 2008, pp. 1270-1275. doi:10.1634/theoncologist.2008-0181

[41] S. Gattenlohner, B. Etschmann, V. Kunzmann, A. Thalheimer, M. Hack, G. Kleber, et al., "Concordance of KRAS/BRAF Mutation Status in Metastatic Colorectal Cancer before and after Anti-EGFR Therapy," Journal of Oncology, Vol. 2009, 2009, Article ID: 831626. doi:10.1155/2009/831626

[42] D. Santini, B. Vincenzi, R. Addeo, C. Garufi, G. Masi, M. Scartozzi, et al., "Cetuximab Rechallenge in Metastatic Colorectal Cancer Patients: How to Come Away from Acquired Resistance?” Annals of Oncology, Vol. 23, No. 9, 2012, pp. 2313-2318. doi:10.1093/annonc/mdr623

[43] J. Metges, J. Raoul, N. Achour, O. Capitain, A. Gourlaouen, J. Ramee, et al., "PANERB study: Panitumumab after Cetuximab-Based Regimen Failure,” Journal of Clinical Oncology, Vol. 28, No. 15, 2010, p. e140000.

[44] R. C. Wadlow, A. F. Hezel, T. A. Abrams, L. S. Blaszkowsky, C. S. Fuchs, M. H. Kulke, et al., "Panitumumab in Patients with KRAS Wild-Type Colorectal Cancer after Progression on Cetuximab,” The Oncologist, Vol. 17, No. 1, 2012, pp. 14, pp. e27-e34.

doi:10.1634/theoncologist.2011-0452

[45] C. Tournigand, T. André, E. Achille, G. Lledo, M. Flesh, D. Mery-Mignard, et al., "FOLFIRI Followed by FOLFOX6 or the Reverse Sequence in Advanced Colorectal Cancer: A Randomized GERCOR Study," Journal of Clinical Oncology, Vol. 22, No. 2, 2004, pp. 229-237. doi:10.1200/JCO.2004.05.113

[46] A. Grothey, D. Sargent, R. M. Goldberg and H.-J. Schmoll, "Survival of Patients with Advanced Colorectal Cancer Improves with the Availability of FluorouracilLeucovorin, Irinotecan, and Oxaliplatin in the Course of Treatment," Journal of Clinical Oncology, Vol. 22, No. 7, 2004, pp. 1209-1214. doi:10.1200/JCO.2004.11.037

[47] B. J, Giantonio, P. J. Catalano, N. J. Meropol, P. J. O’Dwyer, E. P. Mitchell, S. R. Alberts, et al., "Bevacizumab in Combination with Oxaliplatin, Fluorouracil, and Leucovorin (FOLFOX4) for Previously Treated Metastatic Colorectal Cancer: Results from the Eastern Cooperative Oncology Group Study E3200,” Journal of Clinical Oncology, Vol. 25, No. 12, 2007, pp. 1539-1544. doi:10.1200/JCO.2006.09.6305

[48] R. Bianco, R. Rosa, V. Damiano, G. Daniele, T. Gelardi, S. Garofalo, et al., "Vascular Endothelial Growth Factor Receptor-1 Contributes to Resistance to Anti-Epidermal Growth Factor Receptor Drugs in Human Cancer Cells," Clinical Cancer Research, Vol. 14, No. 16, 2008, pp. 5069-5080. doi:10.1158/1078-0432.CCR-07-4905

[49] G. N. Naumov, M. B. Nilsson, T. Cascone, A. Briggs, O, Straume, L. A. Akslen, et al., "Combined Vascular Endothelial Growth Factor Receptor and Epidermal Growth 
Factor Receptor (EGFR) Blockade Inhibits Tumor Growth in Xenograft Models of EGFR Inhibitor Resistance," Clinical Cancer Research, Vol. 15, No. 10, 2009, pp. 3484-3494. doi:10.1158/1078-0432.CCR-08-2904

[50] A. Viloria-Petit, T. Crombet, S. Jothy, D. Hicklin, P. Bohlen, J. M. Schlaeppi, et al., “Acquired Resistance to the Antitumor Effect of Epidermal Growth Factor Receptor-Blocking Antibodies in Vivo," Cancer Research, Vol. 61, No. 13, 2001, pp. 5090-5101.

[51] E. Norguet, L. Dahan, J. Gaudart, M. Gasmi, L. H. Ouafik and J.-F. Seitz, "Cetuximab after Bevacizumab in
Metastatic Colorectal Cancer: Is It the Best Sequence?” Digestive and Liver Disease, Vol. 43, No. 11, 2011, pp. 917-919. doi:10.1016/j.dld.2011.06.002

[52] S. Stintzing, L. Fischer von Weikersthal, T. Decker, U. Vehling-Kaiser, E. Jäger, T. Heintges, et al., "FOLFIRI plus Cetuximab versus FOLFIRI plus Bevacizumab as First-Line Treatment for Patients with Metastatic Colorectal Cancer-Subgroup Analysis of Patients with KRAS: Mutated Tumours in the Randomised German AIO Study KRK-0306,” Annals of Oncology, Vol. 23, No. 7, 2012, pp. 1693-1699. doi:10.1093/annonc/mdr571 